Article

\title{
Composite Wound Dressing Based on Chitin/Chitosan Nanofibers: Processing and Biomedical Applications
}

\author{
Anton S. Shabunin ${ }^{1, *}$, Vladimir E. Yudin ${ }^{1,2}$, Irina P. Dobrovolskaya ${ }^{1,2}$, Evgeny V. Zinovyev ${ }^{3}$, \\ Viktor Zubov ${ }^{3}$, Elena M. Ivan'kova ${ }^{1,2}$ and Pierfrancesco Morganti ${ }^{4}$ \\ 1 Peter the Great St. Petersburg Polytechnic University, Polytechnicheskaya ul. 29, 195251 Saint-Petersburg, \\ Russia; yudinve@gmail.com (V.E.Y.); zair2@mail.ru (I.P.D.); ivelen@mail.ru (E.M.I.) \\ 2 Institute of Macromolecular Compounds, Russian Academy of Sciences, 31 Bolshoy pr. VO, \\ 199004 Saint-Petersburg, Russia \\ 3 Saint-Petersburg State Pediatric Medical University, St. Petersburg, Litovskaya ul. 2y, \\ 194100 Saint-Petersburg, Russia; evz@list.ru (E.V.Z.); viktorzubo@mail.ru (V.Z.) \\ 4 Dermatol Unit, Campania University, L.Vanvitelli, 81100 Naples, Italy; pierfrancesco.morganti@iscd.it \\ * Correspondence: anton-shab@yandex.ru; Tel.: +7-981-840-2652
}

Received: 20 December 2018; Accepted: 26 February 2019; Published: 1 March 2019

\begin{abstract}
An electrospinning technique was used for the preparation of a bilayered wound dressing consisting of a layer of aliphatic copolyamide nanofibers and a layer of composite nanofibers from chitosan and chitin nanofibrils filler. Processed dressings were compared with aliphatic copolyamide nanofiber-based wound dressings and control groups. Experimental studies (in vivo treatment of third-degree burns with this dressing) demonstrated that almost complete (up to 97.8\%) epithelialization of the wound surface had been achieved within 28 days. Planimetric assessment demonstrated a significant acceleration of the wound healing process. Histological analysis of scar tissue indicated the presence of a significant number of microvessels and a low number of infiltrate cells. In the target group, there were no deaths or purulent complications, whereas in the control group these occurred in $25 \%$ and $59.7 \%$ of cases, respectively-and, in the copolyamide group, $0 \%$ and $11 \%$, respectively. The obtained data show the high efficiency of application of the developed composite chitosan-copolyamide wound dressings for the treatment of burn wounds.
\end{abstract}

Keywords: aliphatic copolyamide; chitosan; chitin nanofibrils; electrospinning; wound dressing; composite nanofibers; burns

\section{Introduction}

One of the most critical concerns facing cosmetologists and dermatologists today is wound care. The wound healing process can be significantly slowed down by various factors. Tissue dystrophy, oxidative damage, moisture imbalance in wound, infections and diverse complications in the area of surgical resection, trauma, or burns are the most common. These factors have an especially high impact on epithelialization and granulation processes, which increases rates of scar tissue or other structural defects forming at the wound bed. Currently, there are numerous techniques that intensify the wound healing process. In the majority of these methods, various compositions and structures of wound dressings are used [1-3].

Due to a large number of factors important for successful wound healing, a longlist of requirements is systematically applied to wound dressings. One of the most important points in this list is to ensure the wound surface shape reproduction, the ease of surgical manipulation, and optimal gas and moisture exchange, necessary for vital cell activity. Another important feature of a modern wound 
dressing is atraumaticity for a newly formed epithelium layer, which should not be destroyed during the removal of a dressing from the wound. Finally, a dressing should prevent bacterial contamination. These properties can be achieved by using porous-type films that are obtained by the electrospinning of polymeric solutions. This method allows for the processing of micro- and nanofibers of various polymers with diameters ranging from tens to hundreds of nanometers. Nanofiber-based films are characterized by low density, high porosity, water permeability, and gas permeability [4-7]. These porous films are widely used as matrices for cell technologies. The chemical structure and porosity of these materials facilitate the adhesion of stem or somatic cells onto the fiber surface, and promote metabolic processes that are necessary for efficient cell proliferation and differentiation. Preparation of nanofibers from alcohol-soluble aliphatic copolyamide (CoPA) (copolymer of poly( $\varepsilon$-caprolactam) [-NH- $\left.\left(\mathrm{CH}_{2}\right)_{5}-\mathrm{CO}-\right] n$ and poly(hexamethylenediamineadipinate) $\left.\left[-\mathrm{NH}\left(\mathrm{CH}_{2}\right)_{6} \mathrm{NHCO}\left(\mathrm{CH}_{2}\right) 4 \mathrm{CO}-\right]_{\mathrm{n}}\right)$ is described in [5]. Good adhesion of stem cells and an absence of cytotoxicity were also demonstrated.

Chitosan is another polymer that is widely used in new materials for medical applications. This biocompatible and biodegradable polysaccharide consists of $\beta-(1-4)$-D-glucosamine and N-acetyl-D-glucosamine units [8]. Products of chitosan biodegradation are non-toxic; as decomposition proceeds, these products are involved in the usual metabolic reactions of an organism. However, it is known [8-10] that processing of fibers from chitosan solutions by electrospinning is not easy due to its polyelectrolytic properties. Mostly, water-soluble polymers, e.g. poly(ethylene oxide), poly(vinyl alcohol), methyl cellulose, and poly(vinyl pyrrolidone), are introduced into aqueous solutions of acetic acid for the successful processing of chitosan-based fibers by electrospinning. The addition of these polymers (up to $50 \mathrm{wt} \%$ with respect to the mass of chitosan) has a detrimental effect on the properties of the end product, e.g., leads to an increase in hygroscopicity and a deterioration in mechanical properties. Due to the non-bioresorbability and high molecular mass of some of these polymers, their excretion from the wound and body can be problematic.

It was established that introducing chitin nanofibrils into a chitosan solution significantly increases the rate of nanofiber formation, and the resulting materials have fewer defects. In addition, introducing chitin nanofibrils into chitosan-based composite nanofibers allowed us to reduce the amount of poly(ethylene oxide) (PEO), which is a water-soluble polymer added into a solution in order to facilitate the formation of fibrous structures during electrospinning [11]. Chitin nanofibrils are also bioresorbable and have anti- or proinflammatory activity depending on the size of the fibrils [12]. These facts allow us to propose chitin nanofibrils as a filler for processing chitosan nanofiber materials for medical purposes.

The present work describes the concept of a two-layered composite wound dressing, where an external layer consists of CoPA-based nanofibers and an internal layer consists of composite nanofibers including chitosan and chitin nanofibrils. Nanofibers of non-resorbable CoPA provide the necessary mechanical properties for a wound dressing, facilitate metabolism between a wound and the external environment, and prevent bacterial contamination. The layer of chitosan nanofibers with chitin nanofibrils provides hemostatic and bactericidal activity to the dressing and should be in direct contact with the wound surface. In the process of integration with an active biological medium, gradual resorption of chitosan and chitin nanofibrils occurs. After removing the dressing, the non-resorbable CoPA layer is separated from the wound surface, while the chitin/chitosan layer remains and facilitates the epithelialization of a wound. The aim of the present work was development of the wound dressing based on CoPA nanofibers and composite nanofibers containing chitosan and chitin nanofibrils, as well as in vivo studies of these materials as wound dressings for the treatment of deep skin wounds.

\section{Materials and Methods}

The materials based on CoPA and chitosan nanofibers were obtained from polymeric solutions by electrospinning [5]. The method of processing composite nanofibers from chitosan and fillers (chitin nanofibrils) and the properties of these materials are described in [11]. Films were prepared 
by electrospinning composite chitosan-based nanofibers onto the surface of a porous film made from CoPA nanofibers.

In the preparation of nanofibers by electrospinning, aliphatic copolyamide (Anid OOO, Yekaterinburg, Russia) with a molecular weight $\left(\mathrm{M}_{\mathrm{W}}\right)$ of about $30 \mathrm{kDa}$ was used. In the preparation of composite nanofibers by electrospinning, chitosan with $\mathrm{M}_{\mathrm{w}}=120 \mathrm{kDa}$ and deacetylation degree of $92 \%$ was used (Bioprogress OOO, Voronezh, Russia). Chitin nanofibrils (SRL Mavi Sud, Aprilia, Italy) were used as a filler. The concentration of chitin in the aqueous dispersion was $31.9 \mathrm{mg} / \mathrm{mL}$, the electrical conductivity of the dispersion $(\sigma)$ was equal to $116 \mu \mathrm{S} / \mathrm{cm}$, and the $\mathrm{pH}$ was4.87. Glacial acetic acid was purchased from Vekton ZAO (Saint Petersburg, Russia). PEO with $\mathrm{M}_{\mathrm{w}}=900 \mathrm{kDa}$ was purchased from Union Carbide Corp (Houston, TX, USA). The thickness of CoPA nanofibers layer was 100-150 $\mu \mathrm{m}$, and the thickness of the layer of composite nanofibers was $50 \mu \mathrm{m}$. Electrospinning was performed using a NANON 01 instrument (Ogōri-shi, Japan) at a voltage of $18 \mathrm{kV}$; the distance between electrodes was $0.15 \mathrm{~m}$. The resulting material (size: $10 \mathrm{~cm} \times 5 \mathrm{~cm}$ ) is shown in Figure 1 .

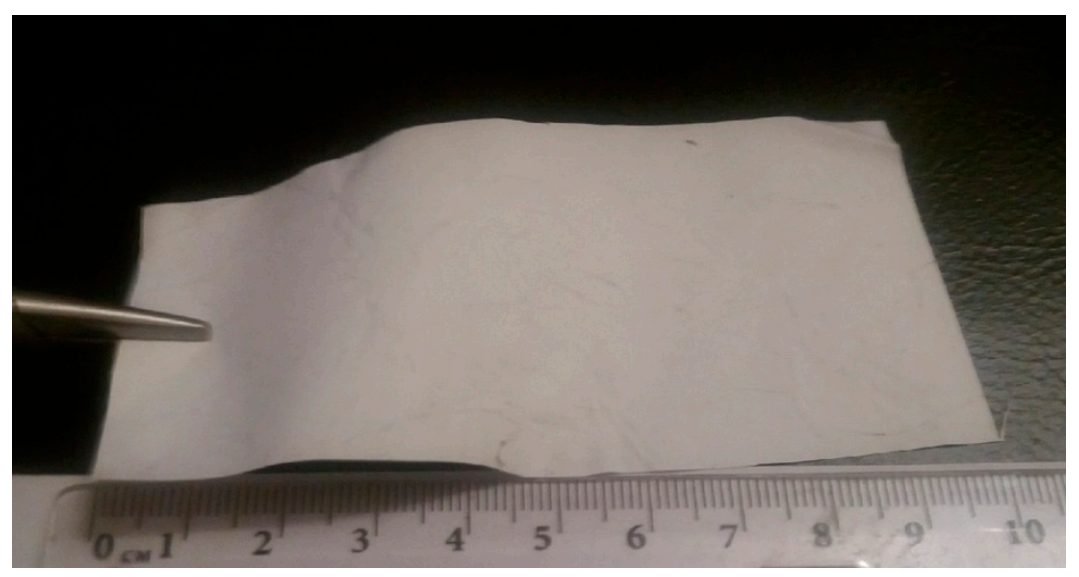

Figure 1. Picture of processed material.

The structure of nanofibers and nanofiber-based materials was studied by scanning electron microscopy.

The measurements were carried out using a Supra 55VP electronic microscope (CarlZeiss, Oberkochen, Germany). The accelerating voltage was $5 \mathrm{kV}$, and samples were sputtered with platinum $(\mathrm{Pt})$. The average values of the diameter of the nanofibers were determined using the AxioVision Rel. Program, micrographs of micro- and nanofibers were used, and averaging was performed on images of 100 fibers.

Twenty-four animals were used in the experiments (Wystar-Kyoto male rats, mass 200-250 g). The animals were divided into three groups (a control group, a CoPA-fibers wound dressing group, and a chitosan-CoPA wound dressing group; eight animals each).Preclinical studies of the processed materials with the use of experimental animals do not require licensing and special permits and should be justified by the decision of the Local Ethics Committee. For ethical reasons, groups of experimental animals included the minimum number of individuals sufficient for obtaining reliable results.

In the experimental groups, after modeling a third-degree burn (ICD 10) $(10 \%$ from the animal surface area) (Figure 2a) and necrectomy and fixation of wound edges (Figure 2b), the wound surface was covered with appropriate dressings. The surface area of the burn was $16 \mathrm{~cm}^{2}$. The animals were observed for four weeks. Observations, planimetric assessment, and biopsy were performed at 7, 14, 21 , and 28 days; morphological studies of biopsy samples were carried out. All manipulations were performed under inhalation anesthesia with diethyl ether and in strict compliance with the provisions of WMA Declaration of Helsinki. 


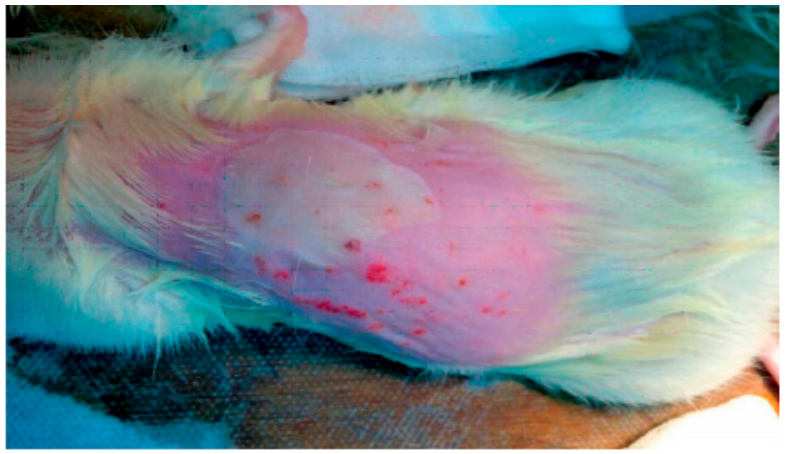

(a)

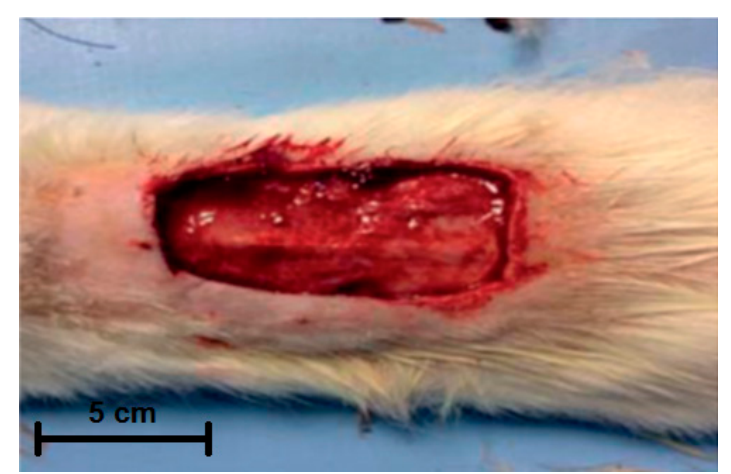

(b)

Figure 2. Wound surface before (a) and after (b) necrectomy.

Planimetric assessment of the wound surface was used to determine the speed and healing acceleration index. The reliability of the wound surface comparison was evaluated by Mann-Whitney criteria. Healing indices were calculated by the following equation [13]:

$$
\left(S-S_{n}\right) \times 100 /(S \times T),
$$

where $S$ is the previous surface area $\left(\mathrm{cm}^{2}\right), S_{n}$ is the actual surface area $\left(\mathrm{cm}^{2}\right)$, and $T$ is the period between observations (days).

Due to the small groups $(N=8)$ and the presence of outliers, neither normal distribution nor q-distribution can be used for a proper reliability assessment. The Mann-Whitney non-parametric method of analysis was chosen to assess the reliability of surface area values difference. Instead of average values, the medians for the samples were analyzed. To describe the variation of samples, interquartile ranges (IQRs) (the difference between the upper and lower quartiles of a sample) are presented. The $p$-value, which is the most common value used in reliability assessments, was determined by Mann-Whitney U-test calculations. Ratios between $p$-values and $\mathrm{u}$-values are tabulated values. If the $p$-value is below the usually agreed alpha risk of $5 \%(0.05)$, the null hypothesis can be rejected and at least one significant difference can be assumed. A null hypothesis is a general statement or default position that there is no difference among select groups.

Tissue samples were fixed in a $10 \%$ solution of neutral formalin in phosphate buffer $(\mathrm{pH}=7.4)$ for $24 \mathrm{~h}$, then treated with ethanol solutions of increasing concentrations $(10 \%, 30 \%, 50 \%, 80 \%$, and 99\%) and embedded in paraffin. Paraffin sections (5 $\mu \mathrm{m}$ thick) were dyed with hematoxylin and eosin (Bio-Optica, Milan, Italy). Microscopy analysis and registration of images were performed with the use of a Leica DM750 light microscope (Wetzlar, Germany).

\section{Results}

\subsection{SEM Microphotographs of Processed Material}

Figure 3 presents microphotographs of the CoPA nanofibers layer and chitosan-based composite nanofibers containing $20 \mathrm{wt} \%$ of chitin nanofibrils layer. It can be seen that the average diameter of CoPA fibers is $600 \mathrm{~nm}$, and the average diameter of chitosan-based composite fibers is $100 \mathrm{~nm}$. 


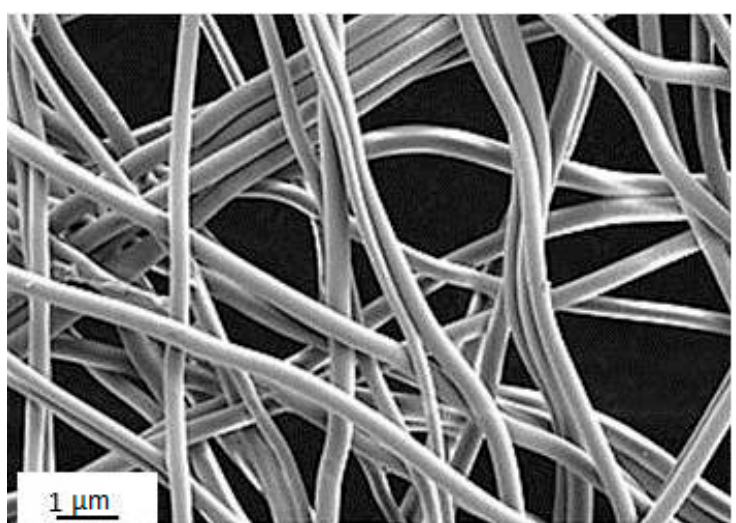

(a)

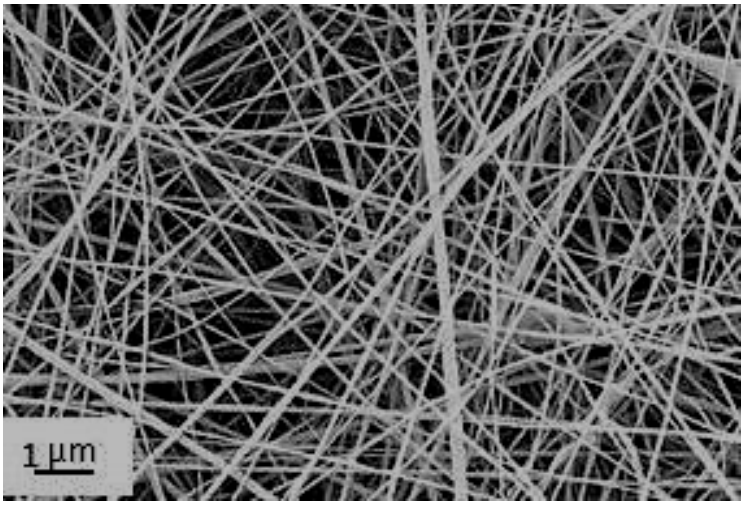

(b)

Figure 3. Microphotographs of CoPA fibers (a) and chitosan-based composite nanofibers containing chitin nanofibrils (b).

\subsection{Observation Results for In Vivo Studies}

Observation of the animals of the control group demonstrated that death occurred in $25 \%$ of cases; purulent complications were registered in $59.7 \%$ of cases (Figure $4 a$ ).

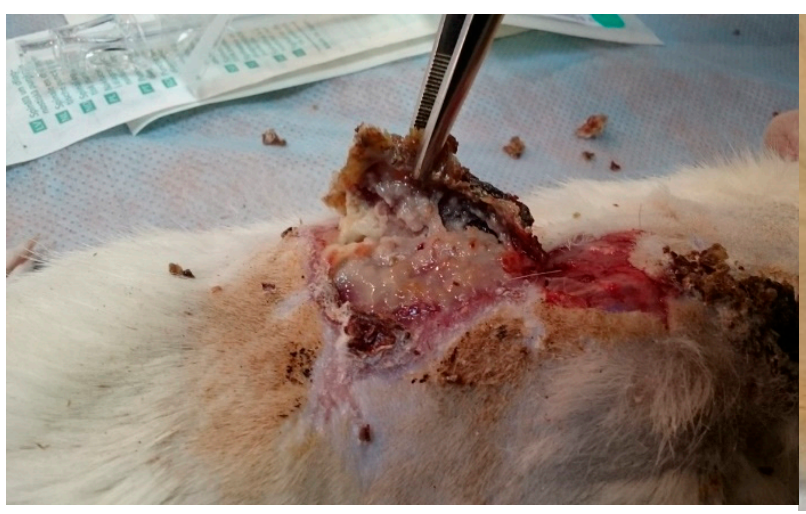

(a)

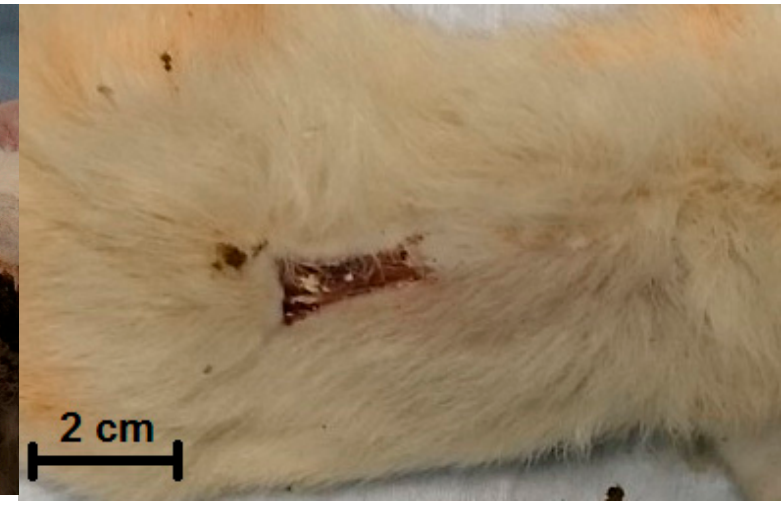

(b)

Figure 4. Visual appearance of the wound surface of an animal from the control group (a) and of an animal after applying the composite wound dressing (b) on the 28th day.

In the experimental group that received treatment in the form of a copolyamide-based wound dressing, no deaths were observed; however, the likelihood of purulent complications was $11 \%$.

In the group of animals treated by the application of a chitosan-CoPA wound dressing, neither fatal outcomes nor purulent complications were observed.

\subsection{Planimetric Assessment}

From Figure 5 and Tables 1 and 2 it can be seen that on the 7 th day no significant difference between the groups was noticed; the statistical difference between the dressing groups and the control group is insignificant $(p>0.05)$. Furthermore, the groups have demonstrated different results. The group of wound dressings based on CoPA nanofibers during the whole treatment does not allow us to make reliable assertions about the differences to the control group, since $p>0.05$.

The comparison of chitosan-copolyamide and control groups allows to assume a significant difference at 14, 21 and 28 days ( $p$-value $<0.05$ ). The chitosan-CoPA group also has a reliable difference at 7,14 , and 21 days, in comparison with the CoPA group. 


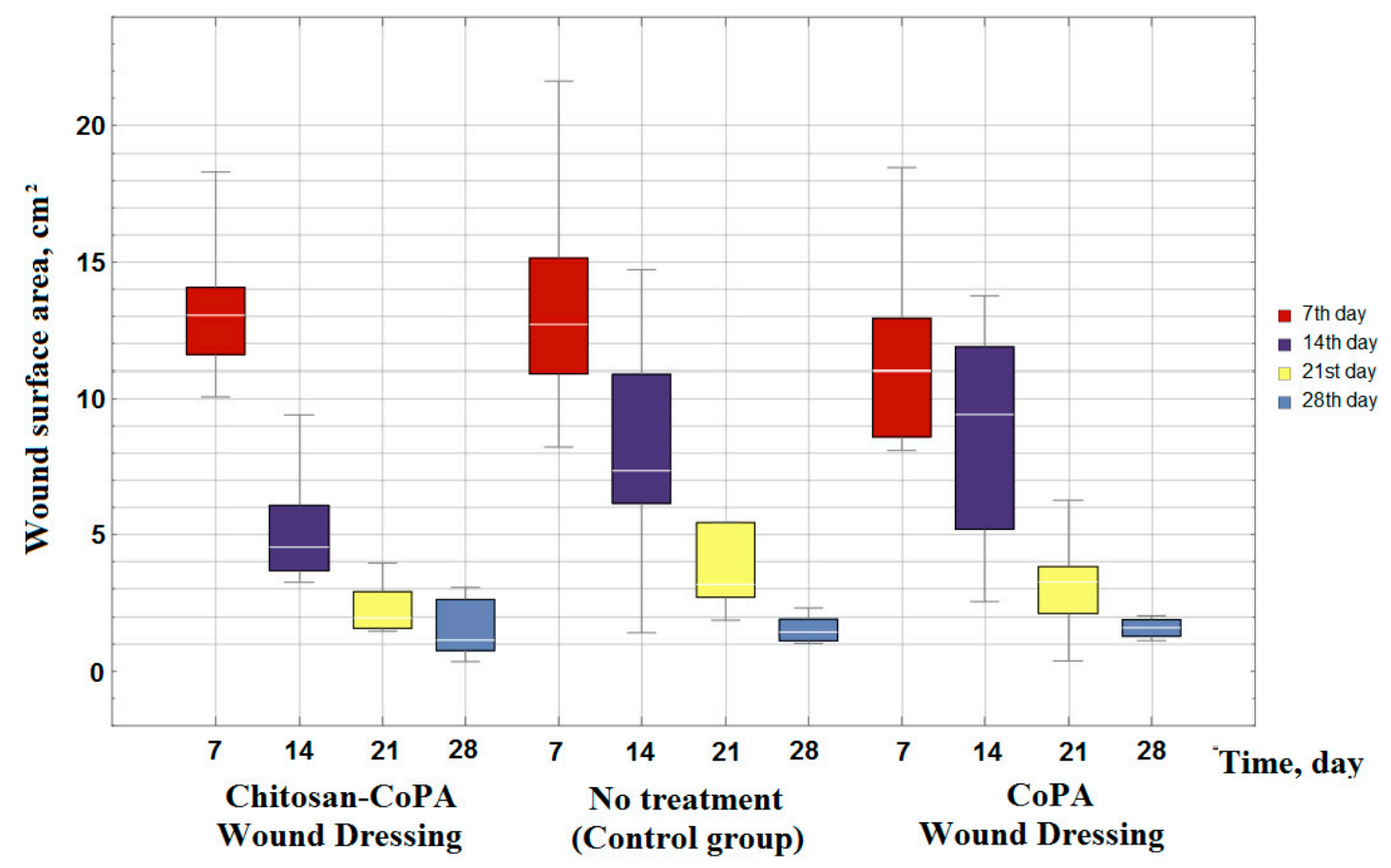

Figure 5. Box-plot of planimetric assessment data of wound surface area after different periods of observation.

Table 1. Surface area planimetric assessment data, including medians and interquartile ranges.

\begin{tabular}{ccccccc}
\hline Group & \multicolumn{2}{c}{ Chitosan-CoPA } & \multicolumn{2}{c}{ CoPA } & \multicolumn{2}{c}{ Control } \\
\hline & Median, $\mathrm{cm}^{2}$ & $\mathrm{IQR}, \mathrm{cm}^{2}$ & Median, $\mathrm{cm}^{2}$ & IQR, $\mathrm{cm}^{2}$ & Median, $\mathrm{cm}^{2}$ & IQR, $\mathrm{cm}^{2}$ \\
7th day & 13.04 & 2.48 & 11.01 & 4.33 & 12.70 & 4.23 \\
14th day & 4.53 & 2.41 & 9.43 & 6.68 & 7.35 & 4.76 \\
21st day & 1.97 & 1.30 & 3.25 & 1.68 & 3.16 & 2.75 \\
28th day & 1.15 & 1.84 & 1.61 & 0.61 & 1.45 & 0.80 \\
\hline
\end{tabular}

Table 2. $P$-values for group comparisons.

\begin{tabular}{cccc}
\hline Groups of Comparison & Chitosan-CoPA / CoPA & Chitosan-CoPA/ Control & CoPA/Control \\
\hline 7th day & 0.05 & 0.83 & 0.15 \\
14th day & 0.04 & 0.03 & 0.73 \\
21st day & 0.08 & 0.04 & 0.77 \\
28th day & 0.28 & 0.05 & 0.71 \\
\hline
\end{tabular}

A graph of the dynamics of the healing indices (Figure 6) shows that the chitosan-CoPA group has a significant increase in value on the 14th day, unlike other groups, in which these values were reached only by the 21st day. Moreover, it is demonstrated that on the 28th day there is no decrease in this dynamic. Thus, it can be argued that in the case of chitosan-copolyamide dressings, tissue regeneration not only begins much earlier than in the other groups, but also has a plateau-like character, while CoPA and control show peak-like dynamics with a maximum on the 21st day. 


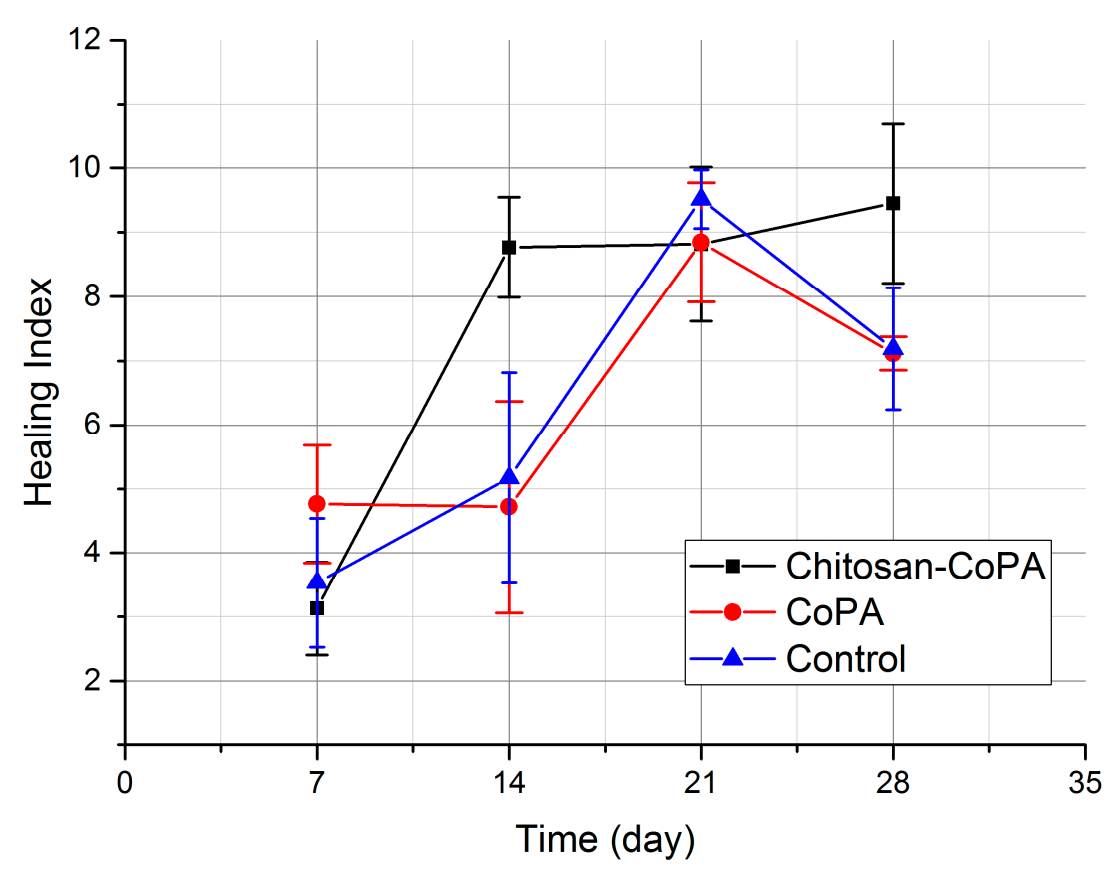

Figure 6. Comparison of healing indices for experimental and control groups.

\subsection{Histological Examination}

Morphological studies showed that, in the animals of the control group, at the scar area, neutrophilous leukocytes prevailed over other infiltrate cells (Figure 7a); maturation of granulation tissue was less pronounced. This granulation tissue covers a rather large area compared to that of the experimental groups. At the same time, in some cases in the chitosan-CoPA experimental group, almost complete epidermization of the wound surface was observed on the 28th day (Figure 2b); the connective tissue occupied a rather small area in comparison to that of the control group. The number of visible microvessels was higher in the chitosan-CoPA group in biopsies on the 14th, 21st, and 28th days (Figure $7 \mathrm{~b}$ ); also, an insignificant number of infiltrate cells was detected at this time in comparison with the CoPA (Figure 7c) and control groups.

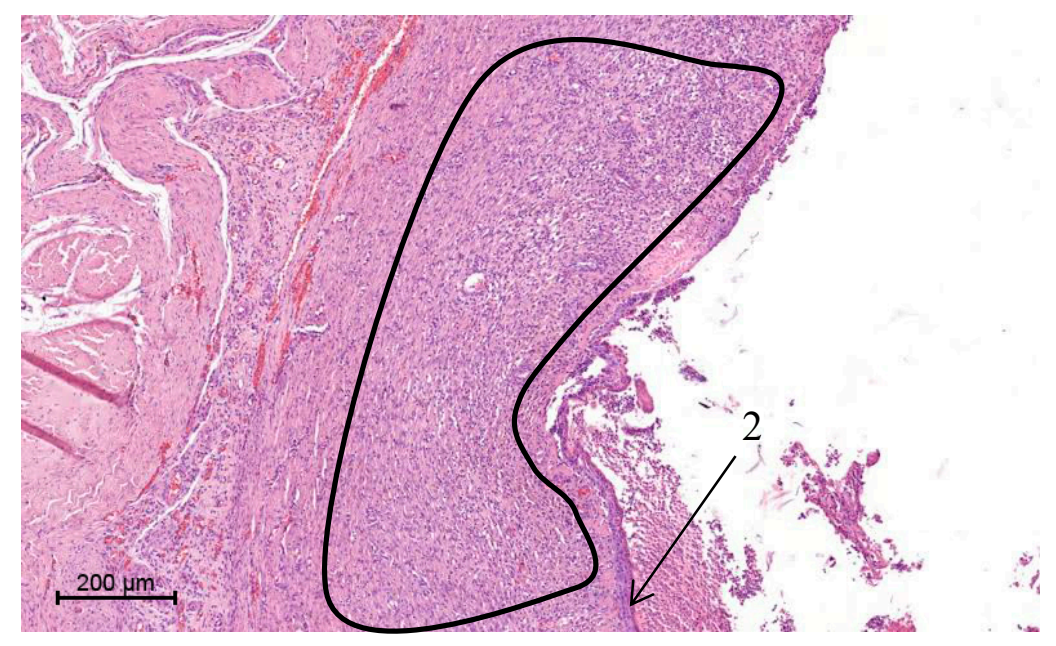

(a)

Figure 7. Cont. 


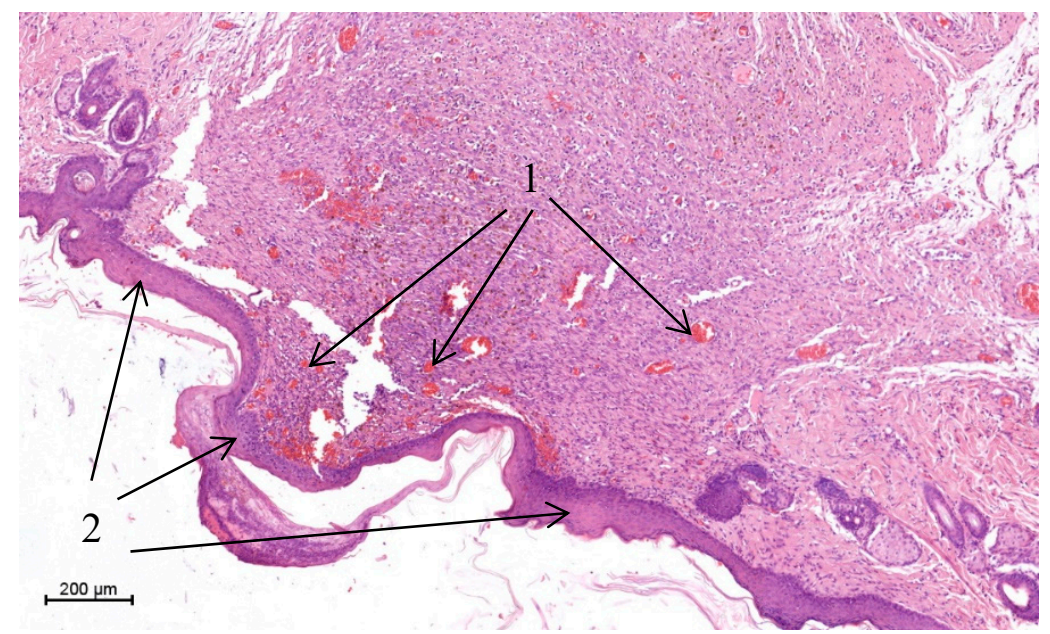

(b)

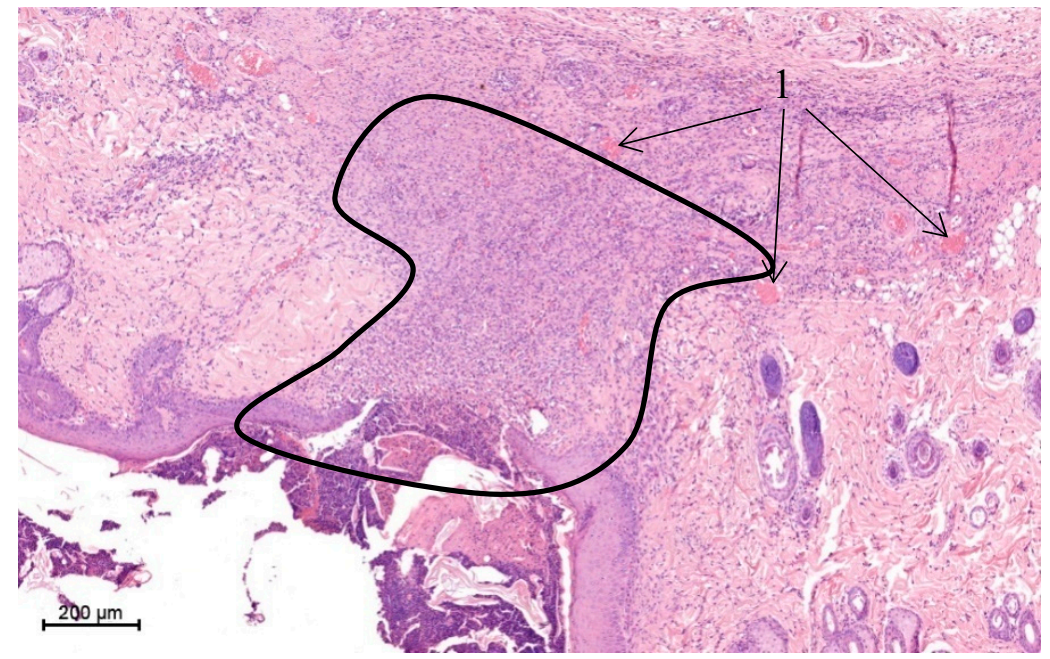

(c)

Figure 7. Histological view of the scar area of a rat from the control (a), chitosan-CoPA (b), and CoPA (c) groups (magnification: $5 \times$ ). The selected areas are neutrophilous leukocytes infiltrated tissues; 1-microvessels; 2-newly formed epithelium.

\section{Discussion}

As is known [1-3], the modern wound dressing should provide gas and vapor exchange between the wound surface and the external environment. This is required for the normal course of cellular processes. The dressing material must have sufficient elasticity to reproduce the relief of the wound surface. Atraumacity is another vitally important characteristic of a modern wound dressing: when the dressing is separated from the wound, the newly formed epithelium layer should not be destroyed. Finally, infection caused by penetration of pathogenic microflora from the external environment dressing should be prevented.

The conducted studies therefore demonstrate that the electrospinning method can be used to process the bilayered composite film material (Figure 1) with a thickness of about 300 microns. The top layer consists of microfibers based on non-resorbable biocompatible synthetic copolyamide. The lower layer in contact with the wound surface consists of composite nanofibers based on the bioresorbable natural polymers chitin and chitosan. The processing of the material is characterized by high stability and does not require special equipment or complex temperature-time regimes. The processed film 
material is characterized by the mechanical properties required for its comfortable manipulations as a wound dressing both in air and in aqueous media.

The features of CoPA microfibers processed by the electrospinning method and the properties of the derived film material are given in [5], which shows that porous films are characterized by relatively high strength and deformation properties in both dry and wet conditions. This material has good hydrophilic attributes, as well as high air and vapor permeability. The optimal transport characteristics of the material required for cellular metabolic processes provide high proliferative activity of mesenchymal stem cells and fibroblasts. Film materials from CoPA, obtained by electrospinning, can be used as matrices for cell cultivation. Furthermore, the porous structure of this material prevents the penetration of pathogens from the external environment onto the surface of the wound. An analysis of the functions of the pore size distribution shows that the materials processed by electrospinning are characterized by bimodal distribution. According to the method of mercury porosimetry, the radius of the mesopore is in the range of 1.3-2.7 $\mu \mathrm{m}$. At the same time, for these materials the presence of large pores with sizes in the tens of $\mu \mathrm{m}$ is also typical. This type of pore structure is clearly demonstrated by SEM micrographs of materials on the basis of nanofibers (Figure 3a).

However, taking into consideration that the wound surface area is dependent on the exposure time (Figure 5), the porous film from the CoPA does not meet the requirements for materials for wound dressings. The area of the wound surface decreases at almost the same rates (Figures 5 and 6) as in the control group, in which no wound dressing was applied. Therefore, to accelerate the wound healing an additional layer of biocompatible polymer, such as chitosan, is essential.

The electrospinning of composite nanofibers based on chitosan and chitin nanofibrils is described in [11], which presents data on the highly proliferative activity of mesenchymal stem cells (MSCs) on composite chitosan matrices. Unlike materials from CoPA, chitosan fibers, films, and sponges under the action of a biologically active medium and the extracellular matrix resorb without adversely affecting the surrounding tissues and organs [14,15].

The resulting composite wound dressings combine good mechanical characteristics, causing good adhesion to the surface of the wound, and the convenience of the performed manipulations, as well as biological characteristics required for successfully accelerating the healing process and minimizing complications. Planimetric assessment data allows us to reliably $(p<0.05)$ state that the wound healing process was significantly accelerated during the treatment by a chitosan-CoPA wound dressing over the entire observation period in comparison with the control group and the CoPA wound dressing group. This can also be confirmed by a comparison of the dynamics of healing indices. Histological examination of biopsy samples shows significant improvement in the quality of the newly formed tissue, along with a decrease in the inflammation and the bactericidal activity of the developed dressings. Also, ease of use and ease of fixing both types of dressings were noted.

The obtained data allow us to confirm the high efficiency of the developed composite chitosan-copolyamide wound dressings for the treatment of burn wounds and create broad prerequisites for the introduction of these dressings in clinical practice.

\section{Patents}

Patent RU 2647609 is a result of this research.

Author Contributions: Data curation, V.Z. and E.M.I.; Formal analysis, A.S.S.; Investigation, A.S.S., V.Z. and E.M.I.; Methodology, I.P.D. and E.V.Z.; Project administration, V.E.Y.; Resources, P.M.; Supervision, V.E.Y., I.P.D. and E.V.Z.; Writing - original draft, A.S.S.

Funding: Russian Science Foundation under contract grant \# 19-73-30003.

Conflicts of Interest: The authors declare no conflict of interest. 


\section{References}

1. Mi, F.L.; Shyu, S.S.; Wu, Y.B.; Lee, S.T.; Shyong, J.Y.; Huang, R.N. Fabrication and characterization of a sponge-like asymmetric chitosan membrane as a wound dressing. Biomaterials 2001, 22, 165-173; [CrossRef]

2. Campbell, T.D.; Wiesmann, W.P.; McCarthy, S.J. Method for Preparing a Compressed Wound Dressing. U.S. Patent 8,313,474, 20 November 2012.

3. Johnson, R.; Tumey, D. Biocompatible Wound Dressing. U.S. Patent 8735644B2, 27 May 2014.

4. Baumgartner, P.K. Electrostatic Spinning of Acrylic Microfibers. J. Coll. Interface Sci. 1971, 36, 71-79. [CrossRef]

5. Dobrovolskaya, I.P.; Popryadukhin, P.V.; Yudin, V.E.; Ivan'kova, E.M.; Elokhovskiy, V.Y.; Weishauptova, Z.; Balik, K. Structure and properties of porous films based on aliphatic copolyamide developed for cellular technologies. J. Mater. Sci. Mater. Med. 2015, 26, 46. [CrossRef] [PubMed]

6. Sill, T.J.; von Recum, H.A. Electrospinning: Applications in drug delivery and tissue engineering. Biomaterials 2008, 29, 1989-2006. [CrossRef] [PubMed]

7. Schiffman, J.D.; Schauer, C.L. A Review Electrospinning of Biopolymer Nanofibers and their Applications. Polym. Rev. 2008, 48, 317-352. [CrossRef]

8. Spasova, M.; Manolova, N.; Paneva, D.; Rashkov, I. Preparation of chitosan-containing nanofibers by electrospinning of chitosan/poly(ethylene oxide) blend solutions. e-Polymers 2004, 56, 1-12. [CrossRef]

9. Li, L.; Hsieh, Y.L. Chitosan bicomponent nanofibers and nanoporous fibers. Carbohydr. Res. 2006, 41, $374-381$. [CrossRef] [PubMed]

10. Zhang, Y.; Huang, X.; Duan, B.; Wu, L.; Li, S.; Yuan, X. Preparation of electrospun chitosan/poly(vinyl alcohol) membranes. Colloid Polym. Sci. 2007, 11, 855-863. [CrossRef]

11. Dobrovolskaya, I.P.; Yudin, V.E.; Popryadukhin, P.V.; Ivan'kova, E.M.; Shabunin, A.S.; Kasatkin, I.A.; Morganti, P. Effect of chitin nanofibrils on electrospinning of chitosan-based composite nanofibers. Carbohydr. Polym. 2018, 194, 260-266. [CrossRef] [PubMed]

12. Morganti, P.; Febo, P.; Cardillo, M.; Donnarumma, G.; Baroni, A. Chitin Nanofibril and Nanolignin: Natural Polymers of Biomedical Interest. J. Clin. Cosmet. Dermatol. 2017, 1. [CrossRef]

13. Fenchin, K.M. Wound Healing [in Russian]; Zdorovie: Kiev, Ukraine, 1979; pp. 66-69, ISBN 978-5458435765.

14. Dobrovolskaya, I.P.; Yudin, V.E.; Popryadukhin, P.V.; Dresvyanina, E.N.; Yudenko, A.N.; Ivan'kova, E.M. In vivo studies of chitosan fiber resorption. J. Appl. Cosmetol. 2015, 33, 81-87.

15. Popryadukhin, P.V.; Yukina, G.Y.; Suslov, D.N.; Dobrovolskaya, I.P.; Ivan'kova, E.M.; Yudin, V.E. Bioresorption of porous three-dimensional chitosan-based materials intended for use in medical surgical cosmetology and tissue engineering. J. Appl. Cosmetol. 2017, 35, 93-104. 